\title{
Percepções da comunidade escolar sobre a implantação do programa unplugged em escolas
}

\author{
Pollyanna Fausta Pimentel de Medeiros ${ }^{1}$, https://orcid.org/0000-0003-1679-5330 \\ Ana Paula Dias Pereira1, http://orcid.org/0000-0001-9944-4004 \\ Daniela Ribeiro Schneider², http://orcid.org/0000-0002-2936-6503 \\ Zila M. Sanchez'1, http://orcid.org/0000-0002-7427-7956
}

\begin{abstract}
Resumo
O objetivo do presente estudo foi investigar as percepções da comunidade escolar sobre o programa Unplugged, implantado pelo Ministério da Saúde em escolas públicas brasileiras de ensino fundamental II. A metodologia utilizada foi a qualitativa e envolveu coleta de dados através de 11 grupos focais de professores $(n=13)$, alunos $(n=54)$ e administradores escolares $(n=11)$ em 8 escolas de 3 cidades brasileiras. Análise de conteúdo permitiu a identificação de 3 eixos temáticos. As facilidades identificadas estiveram relacionadas com o suporte técnico para implantação da metodologia, como a formação e supervisão recebida, além do suporte dos administradores das escolas. As dificuldades apontadas estiveram relacionadas ao tempo das aulas, a obtenção de materiais de suporte para as aulas e ao cumprimento adequado do currículo regular. Como potencialidade, identificou-se a melhoria das relações de convivência. Os resultados aqui alcançados permitem o aprimoramento da implantação do programa Unplugged nas escolas brasileiras.
\end{abstract}

Palavras-chave: Prevenção do abuso de drogas; educação; pesquisa qualitativa.

\section{School community perceptions on implementation of the unplugged program in schools}

\begin{abstract}
The aim of the present study was to investigate the perceptions of the school community about the Unplugged program, implemented by the Ministry of Health in Brazilian public elementary school. The methodology used was qualitative and involved data collection through 11 focus groups of teachers $(n=13)$, students $(n=54)$ and school administrators $(n=11)$ in 8 schools in 3 Brazilian cities. The content analysis allowed the identification of 3 thematic axes. The facilities identified were related to the technical support for the implementation of the methodology, such as the training and supervision received, and the support of the school administrators. The difficulties pointed out were related to class time, obtaining support materials for classes and adequate fulfillment of the regular curriculum. As potentiality, the improvement of the relations of coexistence was identified. The results obtained here allow the improvement of the implementation of the Unplugged program in Brazilian schools.
\end{abstract}

Keywords: Drug abuse prevention; education; qualitative research.

\section{Percepciones de la comunidad escolar sobre la implantación del programa unplugged en escuelas}

\begin{abstract}
Resumen alcanzados permiten el perfeccionamiento de la implantación del programa Unplugged en las escuelas brasileñas.

Palabras clave: Prevención del abuso de drogas; educación; investigación cualitativa.

1 Universidade Federal de São Paulo, Departamento de Medicina Preventiva - São Paulo - SP - Brasil.

2 Universidade Federal de Santa Catarina, Departamento de Psicologia - Florianópolis - SC - Brasil.
\end{abstract}

El objetivo del presente estudio fue investigar las percepciones de la comunidad escolar sobre el programa Unplugged, implantado por el Ministerio de la Salud en escuelas públicas brasileñas de enseñanza primaria II. Se utilizó la metodología cualitativa e involucró recolecta de datos por intermedio de 11 grupos focales de profesores $(n=13)$, alumnos $(n=54)$ y administradores escolares $(n=11)$ en 8 escuelas de 3 ciudades brasileñas. El análisis de contenido permitió la identificación de 3 ejes temáticos. Las facilidades identificadas estuvieron relacionadas con el soporte técnico para implantación de la metodología, como la formación y supervisión recibida, además del soporte de los administradores de las escuelas. Las dificultades apuntadas estuvieron relacionadas al tiempo de las clases, la obtención de materiales de soporte para las clases y al cumplimiento adecuado del currículo regular. Como potencialidad, se identificó la mejora de las relaciones de convivencia. Los resultados aquí 


\section{Introdução}

As pesquisas brasileiras revelam precocidade na idade do primeiro uso de álcool e outras drogas, o que aumenta, consideravelmente, os riscos do uso abusivo e de consequentes danos à saúde de crianças e adolescentes (Sanchez \& cols., 2013; Horta \& cols., 2014). O estudo epidemiológico nacional recente sobre a prevalência de consumo de drogas entre estudantes de 10 a 19 anos em 27 capitas brasileiras apresentou que $60,5 \%$ dos estudantes haviam consumido bebidas alcoólicas na vida, $25,5 \%$ haviam consumido alguma droga ilícita e 16,9\%, tabaco. Esse estudo ressalta a importância e a necessidade da implementação de um programa universal de prevenção ao uso de drogas no ambiente escolar com a finalidade de reduzir a prevalência identificada (Carlini \& cols., 2010).

A prevenção universal destinada a todos os alunos de uma determinada série, independentemente do nível de risco para consumo de drogas (Foxcroft \& Tsertsvadze, 2012; LeNoue \& Riggs, 2016), é, no ambiente escolar, uma das estratégias necessárias para os adolescentes adquirirem habilidades para recusar a oferta de álcool, tabaco e outras drogas (Catalano \& cols., 2012). Estudos que avaliam a eficácia de programas de prevenção ao uso de drogas evidenciam que intervenções preventivas que integram habilidade de vida e influências sociais são mais eficazes no ambiente escolar (Kreeft \& cols., 2009; Faggiano, 2010; Guo, Lee, Liao, \& Huang, 2015), reduzindo a prevalência do consumo de drogas entre os adolescentes através de modificações de atitudes, crenças e percepções normativas sobre o tema "drogas", ou proporcionando o desenvolvimento de competências e habilidades para a recusa ou postergação do primeiro uso (Giannotta, Vigna-Taglianti, Rosaria Galanti, Scatigna, \& Faggiano, 2014).

A escola desempenha papel fundamental na formação de valores, hábitos e estilo de vida dos adolescentes (Foxcroft \& Tsertsvadze, 2012; Guo \& cols., 2015), visto que é um espaço de socialização e de aprendizado em que os adolescentes passam grande parte do tempo. Nesse sentido, é considerado um ambiente promissor para intervenções preventivas por atingir um grande número de adolescentes ao mesmo tempo (Fagen \& Flay, 2009; LeNoue \& Riggs, 2016). Além disso, a prevenção ao uso de drogas no ambiente escolar pode efetivamente complementar as iniciativas sociais, econômicas e políticas preventivas, não sendo uma ação isolada (Spoth, Greenberg, \& Turrisi, 2008).

Apesar do movimento internacional de fortalecimento e expansão da implantação de programas de prevenção baseados em evidências em escolas (Escritório das Nações Unidas sobre Drogas e Crimes [UNODC], 2014), no Brasil a situação é diferente, visto que há ainda lacunas decorrentes da falta de oferta de programas em manuais e baseados em evidências (Pereira, Paes, \& Sanchez, 2016; Pedroso, Abreu, \& Kinoshita, 2015), do despreparo dos professores para lidar com a temática em sala de aula, da disponibilidade de informações corretas sobre drogas (Ferreira, Sanchez, Ribeiro, Oliveira, \& Nappo Ferreira, 2010) e do baixo suporte da gestão da escola (Moreira, Lemos Vóvio, \& De Micheli, 2015).

Em 2003 foi instituída a Política de Atenção Integral aos Usuários de Álcool e Outras Drogas do Ministério da Saúde, que define prevenção como processo de planejamento e implementação de múltiplas estratégias voltadas para a redução dos fatores de vulnerabilidade e risco específicos e fortalecimento dos fatores de proteção (Brasil, 2003). Em 2011, o governo brasileiro instituiu o Programa Crack é Possível Vencer, visando a redução do consumo já instalado e a redução da demanda futura de drogas pela população, colocando, assim, o uso de drogas como prioridade na agenda das políticas públicas sociais, sobretudo no Sistema Único de Saúde - SUS (Decreto n 7.637, 8 de dezembro de 2011).

Nesse sentido, para ampliar as ações de prevenção ao uso de álcool e outras drogas do Programa Crack é Possível Vencer no ambiente escolar, o Ministério da Saúde, em conjunto com o UNODC Brasil (Escritório das Nações Unidas sobre Drogas e Crimes), realizou adaptação de um Programa de Prevenção ao uso de Drogas chamado Unplugged (Sanchez \& cols., 2016).

O Programa Unplugged foi escolhido tendo em vista seus resultados positivos, evidenciados através de ensaio controlado randomizado em oito países europeus, o qual apontou um retardo no início do uso de tabaco, maconha e álcool entre estudantes de 12 a 14 anos das escolas que implantaram o programa, quando comparados a um grupo controle em que o programa não foi implementado (Faggiano \& cols., 2008; Faggiano \& cols., 2010; Gabrhelik \& cols., 2012). O Unplugged é baseado no modelo de Influência Social Global sustentado pelo tripé: 1) promoção de habilidades de vida, 2) informações sobre drogas e 3) pensamento crítico frente às crenças normativas dos educandos que participam das aulas (Sussman, Stacy, Johnson, Pentz, \& Robertson, 2004).

O Unplugged, em seu modelo original, é aplicado em sala de aula pelo próprio professor, treinado pelos desenvolvedores, durante 12 aulas, num período de 3 meses (Kreeft \& cols., 2009). No entanto, para esse programa ser incorporado como uma política pública nacional de prevenção ao uso de drogas, atendendo às demandas da saúde pública e da educação, faz-se necessário avaliar sua capacidade de entrada nas escolas (Das, Salam, Arshad, Finkelstein, \& Bhutta, 2016).

A pesquisa de avaliação de implementação de programas de prevenção ao uso de drogas contribui para o aperfeiçoamento e obtenção de resultados finais favoráveis (Ishaak, Ishaak, Vries, \& van der Wolf, 2014). Incluir na avaliação os atores principais desse processo é uma iniciativa importante para esse tipo estudo (Ringwalt, Vincus, Ennett, Johnson, \& Rohrbache, 2004; Johnson, Hays, Center, \& Daley, 2004). Os professores, alunos e administradores das escolas são considerados agentes essenciais no processo de implementação de prevenção ao uso de drogas nas escolas, se eles não se apropriarem da proposta do programa, se a teoria estiver desvinculada da prática efetiva, o programa 
não resultará na redução do consumo de drogas entre os estudantes (Fagen \& Flay, 2009).

Desenvolver pesquisas com uma abordagem que possibilite outras análises sobre a qualidade da implementação é necessário para compreender algumas características dos programas (Cross, Gottfredson, Wilson, Rorie, \& Connelle, 2010). Nesse sentido, as opiniões e percepções sobre acertos e falhas identificadas pelos envolvidos nos diversos níveis da implantação permitem a adaptação do programa em pontos essenciais para sua inserção adequada no currículo e para a sustentação de novos programas, garantindo a qualidade da intervenção e, por fim, os resultados esperados (Thaker \& cols., 2008).

O presente estudo objetiva investigar a percepção dos envolvidos no processo de implantação do Programa Unplugged para prevenção do uso de drogas nas escolas em sua fase piloto de adaptação para a realidade brasileira. Entende-se que o resultado desta pesquisa contribuirá para a qualificação e entendimento da adaptação de programas escolares de prevenção ao uso de drogas, bem como as potencialidades do Unplugged na prevenção ao uso de drogas em ambiente escolar.

\section{Método}

O presente estudo originou-se de uma pesquisa de avaliação do processo de implantação piloto do Programa Unplugged nas escolas públicas brasileiras (Medeiros, Cruz, Schneider, Sanudo, \& Sanchez, 2016). Caracteriza-se como uma fase de caráter exploratório, constituindo-se numa análise de experiências através de grupo focal, visando à sondagem de possíveis facilidades, dificuldades e potencialidades do processo de implantação do programa. Nessa proposta foi utilizada a metodologia qualitativa, buscando-se compreender e investigar as opiniões dos envolvidos (Flick, 2009; Minayo, Deslandes, Cruz Neto, \& Gomes, 2013). A estratégia de pesquisa adotada contribuiu para uma compreensão aprofundada do fenômeno investigado, da visão de professores, administradores e alunos sobre o Programa Unplugged, de acordo com seus conceitos, revelados de forma aberta em seus discursos orais e escritos (Patton, 2005; Creswell, 2009). Para tanto foram realizados grupos focais com professores, alunos e administradores.

Critérios Consolidados para Reportar Pesquisa Qualitativa (Consolidated Criteria for Reporting Qualitative Research - COREQ) foram utilizados para guiar a estruturação deste artigo.

\section{A Intervenção}

A intervenção ocorreu durante o segundo semestre do ano letivo de 2013 e constituiu-se de um currículo básico estruturado em 12 aulas, as quais foram ministradas semanalmente, com duração média de 1 hora, por professores treinados. O programa é desenvolvido através de técnicas interativas, focado no desenvolvimento de habilidades interpessoais (dinâmicas de grupo, assertividade, resolução de problemas, pensamento criativo e autocontrole), habilidades intrapessoais (comunicação verbal e não verbal, expressão de sentimentos negativos e habilidades de enfrentamento), educação normativa e informações sobre os efeitos do uso de drogas (Vigna-Taglianti \& cols., 2009). O Unplugged é um programa de prevenção universal ao uso de drogas aplicado no ambiente escolar para adolescentes na faixa etária de 11 a 14 anos desenvolvido pelo EU-Dap (Drug Use Prevention Trial - Ensaio de Prevenção de Uso Drogas na Europa). As 12 aulas são guiadas por um manual do professor e um manual do aluno, permitindo replicação em diversos países (Kreeft \& cols., 2009).

O material didático utilizado na intervenção foi traduzido para o português com adaptação linguística, revisão da equivalência gramatical e idiomática e adaptação cultural à realidade brasileira por equipe do Ministério da Saúde, em parceria com o UNODC e os desenvolvedores internacionais. A versão original está disponível no site do EU-Dap (http:// www.eudap.net/Unplugged_HomePage.aspx). O treinamento dos professores foi realizado por profissionais qualificados do Ministério da Saúde que receberam a denominação de "multiplicadoras". Estas, por sua vez, foram treinadas e supervisionadas pelos desenvolvedores europeus do programa e fizeram um acompanhamento sistemático dos professores na aplicação da metodologia (Pedroso \& cols., 2015).

A implantação do Programa Unplugged, no Brasil contou com 6 fases: 1) Planejamento das ações para pactuação entre as políticas de saúde e educação nos estados e municípios; 2) Sensibilização e articulação interfederativa para apresentação da metodologia e seleção das escolas e dos professores; 3) Seleção das multiplicadoras e formação pelos desenvolvedores internacionais da EU-Dap; 4) Treinamento dos professores pelas multiplicadoras; 5) Implantação do programa nas escolas pelos professores e acompanhamento e monitoramento da implantação pelas multiplicadoras; 6) Realização de um estudo de avaliação de processo e de avaliação de resultado por duas universidade brasileiras.

\section{Participantes do Programa}

Participaram do programa 8 escolas, sendo 4 do estado de São Paulo, das cidades São Paulo e São Bernardo do Campo, e 4 do estado de Santa Catarina, da cidade de Florianópolis, totalizando 1.833 alunos, distribuídos em 62 turmas. Foi aplicado por 36 professores (São Paulo: 1.210 alunos, 40 turmas e 15 professores - Santa Catarina: 623 alunos, 22 turmas e 21 professores).

A seleção das escolas participantes foi definida pelas equipes das secretarias de Educação dos municípios envolvidos. Foram selecionadas por sua normalidade, ou seja, não apresentavam nenhum destaque em relação ao consumo de drogas por alunos ou tráfico explícito nos arredores, além de não apresentarem programas formais de prevenção ao uso de drogas em andamento. 
Tabela 1. Participante dos grupos focais divididos por categoria e por estado.

\begin{tabular}{clccc}
\hline $\begin{array}{c}\text { Técnica } \\
\text { Qualitativa }\end{array}$ & \multicolumn{1}{c}{ Participantes } & Amostra & São Paulo & Santa Catarina \\
\hline Grupo Focal & Alunos (07 grupos) & 54 & 21 (02 grupos) & 33 (05 grupos) \\
& Professores (02 grupos) & 13 & 5 (01 grupo) & 8 (01 grupo) \\
& Administradores (02 grupos) & 11 & 8 (01 grupo) & 3 (01 grupo) \\
\hline
\end{tabular}

Quadro 1. Técnica de coleta de dados, participantes, amostra, instrumento e variáveis utilizadas no processo avaliação da execução do programa Unplugged.

\begin{tabular}{|c|c|c|c|c|}
\hline $\begin{array}{c}\text { Técnica } \\
\text { Qualitativa }\end{array}$ & Participantes & Amostra & Instrumento & Variáveis \\
\hline Grupo Focal & $\begin{array}{l}\text { Alunos (07 grupos) } \\
\text { Professores ( } 02 \text { grupos) } \\
\text { Administradores ( } 02 \text { grupos) }\end{array}$ & $\begin{array}{l}54 \\
13 \\
11\end{array}$ & $\begin{array}{l}\text { Roteiro semi- } \\
\text { estruturado }\end{array}$ & $\begin{array}{l}\text { Desenvolvimento de atividade } \\
\text { de prevenção na escola; } \\
\text { Aspectos que fazem o } \\
\text { processo de implementação } \\
\text { difícil ou fácil; Sustentabilidade } \\
\text { do programa; Opiniões sobre } \\
\text { as aulas, Opinião sobre } \\
\text { os resultados percebidos; } \\
\text { Evidência de adaptações na } \\
\text { sala de aula. }\end{array}$ \\
\hline
\end{tabular}

Quadro 2. Descrição das categorias de análise dos dados sobre a percepção do programa Unplugged.

\begin{tabular}{|c|c|}
\hline Categorias & Descrição \\
\hline Facilidades & $\begin{array}{l}\text { Condições técnicas e operacionais para o desenvolvimento } \\
\text { do programa. }\end{array}$ \\
\hline Aspectos analisados & $\begin{array}{l}\text { Formação dos professores, supervisão da equipe e } \\
\text { colaboração da gestão da escola. }\end{array}$ \\
\hline Dificuldades & $\begin{array}{l}\text { Características identificadas pelos envolvidos que } \\
\text { fragilizaram o processo de execução. }\end{array}$ \\
\hline Aspectos analisados & $\begin{array}{l}\text { Tempo para realização do programa, material de apoio, } \\
\text { disciplina e formação dos alunos e o cumprimento do } \\
\text { currículo regular. }\end{array}$ \\
\hline Potencialidades & $\begin{array}{l}\text { Elemento que permitiu uma mudança e que não estava } \\
\text { previsto com a implantação do programa }\end{array}$ \\
\hline Aspectos analisados & $\begin{array}{l}\text { Relações de convivência entre os professores e alunos e } \\
\text { entre os próprios alunos. }\end{array}$ \\
\hline
\end{tabular}

Os professores e turmas que receberam o programa (entre o $6^{\circ}$ e $9^{\circ}$ anos) foram indicados pelos diretores e pelas equipes pedagógicas das escolas. Nas turmas selecionadas, o programa foi aplicado a todos os alunos presentes em sala de aula.

\section{Participantes do Estudo}

Participaram nos grupos focais 54 alunos (7 grupos), 13 professores (2 grupos) e 11 administradores (2 grupos), divididos por categoria e por estado (tabela 1).
Dentre os envolvidos no processo de implantação, recrutaram-se 6 a 10 sujeitos nos três grupos focais: 1) alunos, 2) professores e 3) administradores (diretores e equipe pedagógica), como recomenda Rice e Ezzy (2000), levando em consideração a logística da escola - professores, alunos e administradores que poderiam faltar em suas atividades no dia da coleta de dados.

Os alunos foram convidados a participar do grupo focal voluntariamente pelos diretores, levando-se em conta a participação na maior parte das aulas Unplugged (número de presenças) e capacidade de articular ideias, de acordo com a opinião dos diretores. No que tange aos diretores e 
equipe pedagógica, foram convidados a participar dos grupos focais um diretor e um coordenador pedagógico de cada escola participante.

\section{Instrumentos e Coletas de Dados}

Optou-se pelo grupo focal por ser um método de coleta de dados qualitativos em que os dados são coletados através de interação do grupo sobre o tema escolhido, que contribui na exploração das diferentes formas de comunicação utilizadas pelos participantes na interação. Nesse sentido, o grupo focal tem o potencial de revelar níveis de compreensão de grupo que são inexplorados por outras técnicas de coletas de dados (Doody, Slevin, \& Taggart, 2013). Utilizou-se como instrumento um roteiro com perguntas sobre contexto inicial, a implantação do programa e futuro e sustentabilidade. As questões foram elaboradas permitindo avaliar o significado dos distintos grupos sobre as percepções do Programa. O quadro 1 apresenta o instrumento, a técnica de coleta, número de grupos realizados, participantes envolvidos e temas explorados.

\section{Análises de Dados}

Devido à natureza qualitativa dos dados do discurso, optou-se pela técnica de análise de conteúdo, com o referencial teórico proposto por Bardin. A análise de conteúdo divide-se em três etapas: a) pré-análise; b) exploração do material; e c) tratamento dos resultados, inferência e interpretação (Bardin, 2004).

Cada áudio dos grupos focais foi transcrito na íntegra. Após a transcrição foram realizadas leituras flutuantes dos áudios e exploração dos conteúdos dos grupos focais com base no roteiro semiestruturado. A partir da exploração exaustiva dos conteúdos, foram feitos um desmembramento e um recorte das unidades significativas de respostas discutidas pelos participantes no conjunto de grupos realizados.

Assim, os dados integrados dos grupos focais foram agrupados por temas e, num processo classificatório, foram identificados os seguintes eixos: facilidades, dificuldades e potencialidades do processo de implantação do Programa Unplugged. Esses eixos foram estruturados para permitir a compreensão do contexto em que o programa foi inserido e quais foram os aspectos mais relevantes apontados pelos sujeitos em diferentes níveis de envolvimento e participação do programa. No quadro 2, foi sintetizado a descrição das categorias de análise dos dados sobre a percepção do programa. O programa computadorizado NVivo versão 10 (Gibbs, 2002) foi utilizado como auxiliar em toda a análise qualitativa.

\section{Considerações Éticas}

Todos os procedimentos adotados foram devidamente aprovados pelo Comitê de Ética em Pesquisa da Universidade Federal de São Paulo ( $\left.n^{\circ} 473.498\right)$, e no da Universidade Federal de Santa Catarina $\left(n^{\circ} 711.377\right)$.

\section{Resultados}

Buscou-se identificar percepções sobre a implantação do Programa Unplugged nas escolas. Para tanto, organizaram-se os principais aspectos encontrados, a partir da percepção dos envolvidos, em três eixos temáticos, sintetizados no quadro 3.

Quadro 3. Categorias e sujeitos de análises das opiniões sobre o processo de implantação do Programa Unplugged nas escolas públicas em São Paulo e Santa Catarina.

\begin{tabular}{|c|c|}
\hline Categorias & Sujeitos \\
\hline \multirow{2}{*}{$\begin{array}{l}\text { Facilidades } \\
\text { Formação para aplicação do programa }\end{array}$} & \\
\hline & Professores e Administradores \\
\hline Supervisão das multiplicadoras & Professores e Administradores \\
\hline Colaboração da direção da escola & Professores \\
\hline \multicolumn{2}{|l|}{ Dificuldades } \\
\hline Tempo para realização de uma aula do programa & Professores e Alunos \\
\hline Material para realização das aulas & Professores e Administradores \\
\hline Formação e disciplina dos alunos & Professores, Administradores e Alunos \\
\hline Cumprimento do currículo regular & Professores e Administradores \\
\hline \multicolumn{2}{|l|}{ Potencialidades } \\
\hline Relações de convivência & Professores e Administradores \\
\hline
\end{tabular}




\section{Facilidades}

As facilidades que emergiram dos discursos referiram-se aos aspectos que possibilitaram a aplicação do programa em sala de aula. A partir dos relatos identificaram-se as seguintes categorias: "formação para aplicação do programa", "suporte das multiplicadoras" e "colaboração da direção da escola".

\section{Formação para aplicação do programa}

Os professores e administradores das escolas apontaram, em seus relatos, a oficina de formação do programa como uma etapa fundamental do processo de implantação, que permitiu o desenvolvimento de habilidades para a aplicação em sala de aula, gerando assim maior segurança com o tema e a metodologia do programa.

A formação dá segurança aos professores. Falar sobre drogas é um assunto difícil. A formação foi muito importante $e$ trouxe tranquilidade aos professores. (GF -Administradores)

Eu acho que a formação deu uma mexida nas pessoas, até sobre a possibilidade de você ter respostas. Todo mundo que trata dessa temática [drogas] se sente impotente. Então pensam: "não é comigo porque eu não sei resolver" e, talvez agora, as pessoas tiveram esse conforto de pensar: "não, eu posso participar desse processo, eu consigo fazer alguma coisa (GF - Professores)

\section{Suporte das multiplicadoras}

Os professores e administradores relataram a importância do suporte das multiplicadoras para esclarecimento de dúvidas no processo de aplicação do programa. Este foi um aspecto identificado como facilitador, representado nos seguintes discursos:

$E$ as multiplicadoras, eu acho que são extremamente importantes, fundamentais, na troca de e-mail e quando iam para a escola. Elas passam dicas. (GF - Professor)

Em relação ao apoio, o contato que eu faço sempre que eu tenho alguma dúvida e preciso é com a multiplicadora. Ela tem sido excelente, uma mediadora. Até em alguns momentos difíceis nossos de estresse ela está lá mediando. (GF -Administradores)

\section{Colaboração da direção da escola}

A colaboração da direção da escola foi sinalizada como uma facilidade para implantação do programa, sendo considerada pelos professores como um incentivo necessário ao desenvolvimento do programa.
A minha coordenadora e o diretor sempre estavam lá para me ajudar. Quando eu precisava dar duas aulas seguidas, a coordenadora dizia: tudo bem, a sua outra turma [aquela que teria a aula seguinte com a professora] eu coloco no pátio ou vejo outro professor [para substituir]. (GF - Professor)

Quanto ao apoio do colégio, foi muito tranquilo, inclusive o diretor e a equipe pedagógica toda fez a formação com a gente. Estavam a par da situação, foi bem tranquilo, em tudo que foi possível eles apoiaram. (GF - Professor)

\section{Dificuldades}

As dificuldades de implantação foram apontadas com ênfase pelos professores, tendo em vista que foram eles que aplicaram a metodologia em sala de aula. Identificaram-se obstáculos relativos ao "tempo para realização das aulas", "período de duração do programa", "material de apoio para realização das atividades", "formação acadêmica e disciplina dos alunos" e "cumprimento do currículo regular".

\section{Tempo para realização de uma aula do programa}

A insuficiência de tempo para o desenvolvimento adequado das atividades previstas no Programa Unplugged foi descrita como a principal limitação do programa na opinião dos professores e alunos, uma vez que na duração de uma hora-aula (50 minutos em São Paulo, 45 minutos em Santa Catarina) foi difícil o cumprimento de todas as atividades previstas no manual do programa, para cada uma das aulas. Tal percepção pode ser notada nos discursos a seguir:

... o cálculo que se fez de aplicação de cada atividade, 20 minutos para essa atividade, 15 para aquela outra, 30 para aquela outra, sei lá, ele é totalmente fora da realidade de uma sala com 35 alunos. (GF - Professor)

Essa questão de aplicar em 45 minutos, já conversei várias vezes com a multiplicadora, é totalmente inviável. (GF Professor)

... deveria ter mais tempo para a aula, teve uma aula que a gente começou, ai o sinal bateu e a gente teve que terminar na outra aula [aula seguinte]. Devia assim, ter uma aula especifica para fazer o Unplugged, entendeu? (GF - Aluno)

\section{Material para realização das aulas}

A preparação do material para a realização das atividades das aulas foi relatada por administradores e professores como sendo uma dificuldade no processo de implantação do programa. A principal crítica, segundo os professores, foi a falta de apoio dos administradores na distribuição e oferta 
dos materiais de suporte para as aulas propostas no manual distribuído pelo Ministério da Saúde.

...é muito complicado, não tem material suficiente. Se eu soubesse que aqui na escola não tinha, eu tinha comprado. Então tudo isso atrapalha. (GF - Professor)

Na minha escola a equipe [pedagógica] é que ficou com o material, no caso, as cartolinas não foram nem distribuídas para nós. (GF - Professor)

O preparo das aulas também [gera dificuldades]. Tem um trabalho que o professor precisa fazer que é além da demanda desse professor. (GF - Administradores)

\section{Formação e disciplina dos alunos}

Outra das dificuldades apontadas no processo de implantação do programa, nos relatos dos professores e administradores, foi a condição de aprendizagem e indisciplina de alguns alunos, o que acarretou dificuldades na aplicação do programa. A baixa qualidade do letramento dos alunos segundo os professores que participaram do estudo comprometeu a compreensão do conteúdo e, consequentemente, o desenvolvimento adequado das atividades que solicitavam a utilização dos textos escritos para suscitar reflexão e envolvimento com o tema. A falta de disciplina foi percebida no relato dos alunos, da mesma forma, para eles compromete a implantação do programa, pois os alunos acabam tumultuando as aulas e impedindo o professor de cumprir a aplicação do conteúdo planejado.

Também aparece a dificuldade de escrita dos nossos alunos. Os nossos alunos não sabem escrever. O sexto ano tem alguns alunos analfabetos quase. Tem aquelas perguntas pra eles responderem, se deixar no tempo dele, ele vai levar um tempo enorme pra responder. (GF - Professor)

Alguns tem até dificuldade de leitura. O próprio material exigia mais. (GF -Administradores)

A professora estava tentando explicar a aula, pedia silêncio, pedia para eles parar [ficarem quietos] e eles não paravam. (GF - Alunos)

\section{Cumprimento do currículo escolar}

Por fim, destacou-se ainda como dificuldade séria no processo de implantação o comprometimento de carga horária regular para cumprir o conteúdo curricular da disciplina do professor que ministrou o Unplugged, visto que aulas curriculares de disciplinas como Português, Matemática e Ciências foram substituídas por aulas do Unplugged. Sem reposição da carga horária perdida pela disciplina do professor responsável, o conteúdo curricular ficou comprometido. Assim, as disciplinas curriculares passaram a ser dadas de maneira mais superficial.

Sem contar que o conteúdo [da matéria] fica super atrasado. Que nem, eu sou Professor de Matemática, tenho seis aulas por semana [por turma] e o que acontece? Foi meio semestre que a gente teve que aplicar [Unplugged] (GF - Professor)

Percebo que eles estão um pouco sobrecarregados por que junto a isso eles precisam cumprir o currículo e o tempo foi curto. (GF - Administradores)

\section{Potencialidades}

De acordo com os resultados analisados, todos os envolvidos na implantação do programa apontaram que o programa apresentou potencialidades no que se refere à melhora nas relações de convivência entre os envolvidos (professores e alunos).

\section{Relações de convivência}

Foram enfatizadas melhorias nas relações interpessoais que se estabeleceram durante a implantação do programa. De acordo com os discursos, o programa proporcionou melhora no relacionamento entre os alunos e aproximação de alunos e professores, fortalecendo vínculos e melhorando as relações de confiança entre eles. Professores que passaram a compreender melhor seus alunos, grupos que passaram a incluir alunos excluídos e a percepção de amizade desenvolvida durante o projeto são temas que emergiram dos discursos.

...eu ganhei muito com o Unplugged, eu chego a dizer que no meu lado pessoal de lidar com os problemas, eu ganhei muito. Primeiro eu consegui ter uma visão deles [alunos], do coletivo da sala, o que para mim é muito importante. Eu consegui fazer alguns interagirem, o que é importante. (GF - Professor)

Das duas turmas que são minhas eu também percebi uma mudança. Eles [alunos] se uniram muito. Tem uma aluna que ela é especial e ela acabou fazendo parte dessa aula e todos começaram a se socializar com ela... Eles se uniram muito. (GF - Professor)

Melhorou o relacionamento entre eles [alunos], entre os professores também. (GF - Administradores)

\section{Discussão}

Este estudo demonstrou aspectos relevantes da implantação do Programa Unplugged no ambiente escolar 
na perspectiva de professores, alunos e administradores. Evidenciou, através dos resultados do grupo focal, que apesar da potencialidade do programa e das facilidades de implantação, adaptações relacionadas às dificuldades se fazem necessárias para o programa vir a se tornar uma política pública.

Pesquisas internacionais reforçam a importância de se estudar a implantação de programas de prevenção e defendem investimentos em pesquisas avaliativas para exame da implementação (Domitrovich \& cols., 2008; Durlak, 2015), identificando fatores que podem ser relacionados com a qualidade de execução, investigando o que deu certo e de fato funciona, procurando estabelecer um melhor entendimento na consolidação de programas educacionais no campo da prevenção ao uso de drogas (Payne, Gottfredson, \& Gottfredson, 2006; Forman, Olin, Hoagwood, Crowe, \& Saka, 2009; Catalano \& cols., 2012).

Os resultados obtidos no presente estudo identificaram que as facilidades da implantação do Programa Unplugged estiveram relacionadas com a capacidade de suporte técnico ofertado pelo Ministério da Saúde, formação para aplicação do programa e colaboração da direção da escola. Considera-se que esse, de acordo com as análises realizadas, foi uma estratégia importante para a aceitação da execução do programa e o alcance dos objetivos propostos, e que ele favoreceu a aceitação para implantação do programa de prevenção ao uso de drogas nas escolas, influenciando e operando no micro e macroprocessos de tomada de decisões relacionadas à intervenção nas escolas.

As dificuldades encontradas perpassaram pela inserção do programa na escola. Os administradores, alunos e professores identificaram como dificuldades aspectos relacionados ao tempo para realização de uma aula do programa, ao material para realização das aulas, o baixo letramento e disciplina dos alunos e ao cumprimento do currículo escolar. Um estudo realizado no Havaí (EUA), sobre a inserção de um programa de prevenção ao tabagismo para estudantes, identificou as mesmas barreiras, do ponto de vista organizacional, da inclusão do programa nas escolas (Sy \& Glanz, 2008).

Estudos reforçam uma tendência de abordagens participativas entre os envolvidos no desenvolvimento das intervenções (Malloy \& cols, 2015; Pas, Waasdorp, \& Bradshaw, 2015), possibilitando que as áreas responsáveis pelo programa obtenham retorno sobre os desafios no processo de implementação, para que mudanças sejam feitas na execução e na integração com o currículo escolar, com o objetivo de manter a fidelidade dos programas (Saunders, Evans, \& Joshi, 2005; Sy \& Glanz, 2008; Rajan \& Basch, 2012). No caso do Unplugged, os resultados do presente estudo evidenciam a necessidade de rever alguns processos, para melhorar o processo de implantação pois esses podem dificultar sua aplicação em anos vindouros: quando um professor sente-se angustiado por não conseguir completar seu currículo regular, provavelmente decidirá por aplicar as aulas do Unplugged de maneira mais rápida, para que sobre tempo para ministrar as aulas regulares da disciplina para as quais foi contratado.
Outro aspecto preocupante diz respeito à falta de apoio da gestão escolar no fornecimento de material pedagógico e suporte aos professores. De acordo com as diretrizes internacionais sobre prevenção do uso de drogas publicadas pelo UNODC, a prevenção ao uso de drogas deve ser realizada em um programa que tenha a adesão e implicação do corpo docente e da gestão escolar, assim como da família e da comunidade escolar, utilizando métodos interativos e proporcionando oportunidade de praticar e aprender uma variedade de habilidades pessoais e sociais (UNODC, 2014). O Programa Unplugged oferece essa proposta (Faggiano \& cols., 2008; Kreeft \& cols., 2009; Faggiano \& cols., 2010).- Em algumas escolas, quando ocorreu apoio durante o processo, esse foi reconhecido como imprescindível na implantação, por outro lado, quando ocorreu um distanciamento dos administradores, isso repercutiu na aplicação do programa em sala de aula, conforme percepção dos professores.

Em um estudo qualitativo publicado sobre a avaliação do processo da implantação do Programa Unplugged na República Tcheca (Jurystová \& Miovský, 2010), os autores também encontraram evidências da importância do apoio organizacional. Essa similaridade com a percepção dos sujeitos envolvidos na implantação no Brasil demonstra que em ambos os contextos de inserção do Programa Unplugged se faz necessário um conjunto de ações que precisam ser estabelecidas e adaptadas ao processo de trabalho na escola, as quais não dependem apenas do oferecimento do manual impresso pelo Ministério da Saúde.

Um dos principais achados do estudo, listado no campo das potencialidades do programa e que ainda não havia sido descrito em outros estudos publicados sobre o Unplugged foi a melhoria nas relações de convivência observadas entre professores-alunos e entre alunos-alunos a partir da metologia aplicada em sala de aula. Esse resultado foi idenficado nos gupos focais de professores e administradores. Nesse sentido, além dos desfechos tradicionais sobre a diminuição do uso de drogas já descritos em diversos estudos internacionais (Faggiano \& cols., 2008; Kreeft \& cols., 2009; Faggiano \& cols., 2010), o indicativo de melhora do ambiente escolar no resultado da pesquisa aqui no Brasil surge como potencial desfecho secundário no campo da Saúde e como desfecho primário no campo da Educação, o qual deve agora ser testado em ensaio controlado randomizado.

Apesar dos dados relevantes que este estudo apresenta, há limitações que devem ser levadas em consideração, a começar pelo fato de as escolas participantes do estudo não terem sido randomizadas mas indicadas pelas secretarias de Educação das cidades envolvidas. Além disso, a definição dos alunos para o grupo focal foi feita pela própria equipe da escola, o que pode ter comprometido a representação de todos os perfis dos alunos. Outra limitação do estudo é a impossibilidade de se generalizar seus achados para além do contexto pesquisado. No entanto, a disseminação de pesquisas dessa natureza constitui uma importante contribuição acerca da implantação do Programa Unplugged no Brasil. 
Os resultados apontados relacionam-se com alguns desafios importantes vinculados à realidade da escola, o que reforça a complexidade do processo de trabalho no desenvolvimento da consolidação de temas transversais junto aos profissionais na política educacional brasileira. Este estudo avança no sentido de considerar a percepção dos envolvidos, sobretudo dos professores e alunos. A escola é um espaço institucional, social e político onde se dá o processo educativo e de formação de crianças e adolescentes. Nesse contexto, tem-se de maneira bastante oportuna a possibilidade de influenciar na formação de hábitos e desenvolvimento de habilidades para a vida dos alunos (Saunders \& cols., 2005; Moreira, Silveira, \& Andreoli, 2006; Sy \& Glanz, 2008; Kreeft \& cols., 2009; Ferreira \& cols., 2010; Vadrucci \& cols., 2015).

\section{Considerações Finais}

O presente estudo evidenciou elementos do Programa Unplugged adaptado ao contexto brasileiro, a partir da percepção dos atores envolvidos. Os resultados auxiliam a compreensão das necessidades de adaptações logísticas e organizacionais do Unplugged nas escolas públicas brasileiras. De modo geral, os resultados proporcionaram reflexões pertinentes, que apontam possibilidades concretas para a mudança do paradigma vigente de organização do processo de trabalho na área da Educação.

A percepção da comunidade escolar submetida ao Programa Unplugged indicou, pelo resultado da pesquisa, que a lógica da prevenção ainda não integra o cotidiano e o planejamento de grande parte das escolas públicas, conforme descrito na Política de Atenção Integral aos Usuários de Álcool e Outras Drogas. Percebeu-se que a Prevenção ao Uso de Drogas só será efetiva, no contexto brasileiro, com apoio e estímulo institucionais, compartilhamento e envolvimento dos professores, administradores e participação dos alunos em sala de aula, com adaptações curriculares que não comprometam o ensino regular. Em consonância com esse processo de construção e instrumentalização, para a consolidação de estratégias de qualificação dos projetos político-pedagógicos das ações de prevenção ao uso de drogas nas escolas considera-se necessário institucionalizar e incorporar pesquisas avaliativas que acompanhem e subsidiem a implantação de programas prevenção nas escolas brasileiras.

\section{Referências}

Bardin, L. (2004). Análise de conteúodo (3ª ed.). Lisboa: Edições 70.

Brasil. Ministério da Saúde. (2003). Política de Atenção Integral aos usuários de álcool e outras drogas. Brasil: Ministério da Saúde.

Carlini, E.L.A.; Noto, A.R.; Sanchez, Z.M.; Carlini, C.M.A.; Locatelli, D.P.; Abeid, L.R.; Amato, T.C.; Opaleye, E.S.; Tondowski, C.S.; Moura, Y. G. (2010). VI Levantamento Nacional sobre o Consumo de Drogas Psicotrópicas entre Estudantes do Ensino Fundamental e Médio das Redes Pública e Privada de Ensino nas 27 Capitais Brasileiras. São Paulo: SENAD - Secretaria Nacional de Políticas sobre Drogas.

Catalano, R.F.; Fagan, A.A.; Gavin, L.E.; Greenberg, M.T.; Irwin, C.E.; Ross, D.A.; Shek, D.T. (2012). Worldwide application of prevention science in adolescent health. Lancet, 379(9826), 1653-1664.

Creswell, J. (2009). Research design: Qualitative, quantitative and mixed methods approaches ( $3^{\mathrm{a}}$ ed.). EUA: Sage Publications.

Cross, A.B.; Gottfredson, D.C.; Wilson, D.M.; Rorie, M.; Connell, N. (2010). Implementation quality and positive experiences in afterschool programs. Am J Community Psychol, 45(3-4), 370-380.

Das, J.K.; Salam, R.A.; Arshad, A.; Finkelstein, Y.; Bhutta, Z.A. (2016). Interventions for Adolescent Substance Abuse: An Overview of Systematic Reviews. J Adolesc Health, 59(4S), S61-S75.

Decreto $n^{\circ}$ 7.637, de 8 de dezembro de 2011 (2011, 8 de dezembro). Altera o Decreto no 7.179, de 20 de maio de 2010, que institui o Plano Integrado de Enfrentamento ao Crack e outras Drogas. Brasília: Presidência da República.

Doody, O.; Slevin, E.; Taggart, L. (2013). Focus group interviews part 3: Analysis. British Journal of Nursing, 22(5), 266-9.

Domitrovich, C.E.; Bradshaw, C.P.; Poduska, J. M., Hoagwood, K., Buckley, J. A., Olin, S., ... \& lalongo, N. S. (2008). Maximizing the implementation quality of evidence-based preventive interventions in schools: A conceptual framework. Advances in School Mental Health Promotion, 1(3), 6-28.

Durlak, J.A. (2015). Studying program implementation is not easy but it is essential. Prevention Science, 16(8), 1123-1127.

Escritório das Nações Unidas sobre Drogas e Crimes [UNODC] (2014). Normas Internacionais Sobre a Prevenção do uso de Drogas. Brasília: UNODC.

Fagen, M.C.; Flay, B.R. (2009). Sustaining a school-based prevention program: results from the Aban Aya Sustainability Project. Health Educ Behav, 36(1), 9-23.

Faggiano, F. (2010). Prevention of substance abuse: randomised or observational evaluation is absolutely needed. Adicciones, 22(1), $11-14$.

Faggiano, F.; Galanti, M.R.; Bohrn, K.; Burkhart, G.; Vigna-Taglianti, F.; Cuomo, L.; Group, E. (2008). The effectiveness of a school-based substance abuse prevention program: EU-Dap cluster randomised controlled trial. American Journal of Preventive Medicine, 47(5), 537-543.

Faggiano, F.; Vigna-Taglianti, F.; Burkhart, G.; Bohrn, K.; Cuomo, L.; Gregori, D.; Group, E.D.S. (2010). The effectiveness of a schoolbased substance abuse prevention program: 18-month follow-up 
of the EU-Dap cluster randomized controlled trial. Drug Alcohol Depend, 108(1-2), 56-64.

Ferreira, T.C.D.; Sanchez, Z.M.; Ribeiro, L.A.; Oliveira, L.G.; Nappo, S.A. (2010). Perceptions and attitudes among public school teachers towards the topic of drugs. Interface-Comunicação, Saúde, Educação, 14(34), 551-562.

Flick, U. (2009). Desenho da pesquisa qualitativa: Coleção Pesquisa qualitativa: Bookman Editora.

Forman, S.G.; Olin, S.S.; Hoagwood, K.E.; Crowe, M.; Saka, N. (2009). Evidence-based interventions in schools: Developers' views of implementation barriers and facilitators. School Mental Health, 1(1), 26-36.

Foxcroft, D.R.; Tsertsvadze, A. (2012). Universal alcohol misuse prevention programmes for children and adolescents: Cochrane systematic reviews. Perspect Public Health, 132(3), 128-134.

Gabrhelik, R.; Duncan, A.; Miovsky, M.; Furr-Holden, C.D.; Stastna, L.; Jurystova, L. (2012). “Unplugged”: a school-based randomized control trial to prevent and reduce adolescent substance use in the Czech Republic. Drug Alcohol Depend, 124(1-2), 79-87.

Giannotta, F.; Vigna-Taglianti, F.; Rosaria Galanti, M.; Scatigna, M.; Faggiano, F. (2014). Short-term mediating factors of a schoolbased intervention to prevent youth substance use in Europe. Journal of Adolescent Health, 54(5), 565-573.

Gibbs, G. (2002). Qualitative data analysis: Explorations with NVivo (Understanding social research): Buckingham: Open University Press.

Guo, J.L.; Lee, T.C.; Liao, J.Y.; Huang, C.M. (2015). Prevention of illicit drug use through a school-based program: results of a longitudinal, cluster-randomized controlled trial. Journal of Adolescent Health, 56(3), 314-322.

Horta, R.L.; Horta, B.L., Costa, A.W.; Prado, R.R.; Oliveira-Campos, M.; Malta, D.C. (2014). Lifetime use of illicit drugs and associated factors among Brazilian schoolchildren, National Adolescent School-based Health Survey (PeNSE 2012). Revista Brasileira de Epidemiologia, 17(1), 31-45.

Ishaak, F.; Vries, N.K.; van der Wolf, K. (2014). Test implementation of a school-oriented drug prevention program "Study without Drugs": pre- and post-testing for effectiveness. BMC Public Health, 14, 590.

Johnson, K.; Hays, C.; Center, H.; Daley, C. (2004). Building capacity and sustainable prevention innovations: A sustainability planning model. Evaluation and Program Planning, 27(2), 135-149.

Jurystová, L.; Miovský, M. (2010). Vybrané aspekty organizační a odborné podpory pedagogů při realizaci metodiky Unplugged ve školách: výsledky evaluace implementace projektu EUDAP. Adiktologie,(10), 3, 146-153.
Kreeft, P.V.D.; Wiborg, G.; Galanti, M.R.; Siliquini, R.; Bohrn, K.; Scatigna, M.; Faggiano, F. (2009). 'Unplugged': A new European school programme against substance abuse. Drugs: Education, Prevention, and Policy, 16(2), 167-181.

LeNoue, S.R.; Riggs, P.D. (2016). Substance Abuse Prevention. Child and Adolescent Psychiatric Clinics of North America, 25(2), 297305.

Malloy, M.; Acock, A.; DuBois, D.L.; Vuchinich, S.; Silverthorn, N.; Ji, P.; Flay, B. R. (2015). Teachers' perceptions of school organizational climate as predictors of dosage and quality of implementation of a social-emotional and character development program. Prevention Science, 16(8), 1086-1095.

Medeiros, P.F.; Cruz, J.I.; Schneider, D.R.; Sanudo, A.; Sanchez, Z. M. (2016). Process evaluation of the implementation of the Unplugged Program for drug use prevention in Brazilian schools. Substance abuse treatment, prevention, and policy, 11(1), 1-11.

Minayo, M.C.S.; Deslandes, S.F.; Cruz Neto, O.; Gomes, R. (2013). Pesquisa social: teoria, método e criatividade: Vozes.

Moreira, F.G.; Silveira, D.X.; Andreoli, S. B. (2006). Redução de danos do uso indevido de drogas no contexto da escola promotora de saúde. Ciência \& Saúde Coletiva, 11(3), 807-816.

Moreira, A.; Lemos Vóvio, C.; De Micheli, D. (2015). Prevenção ao consumo abusivo de drogas na escola: desafios e possibilidades para a atuação do educador. Educação e Pesquisa, 41(1).

Patton, M.Q. (2005). Qualitative research: Wiley Online Library.

Payne, A.A.; Gottfredson, D.C.; Gottfredson, G.D. (2006). School predictors of the intensity of implementation of school-based prevention programs: results from a national study. Prevention Science, 7(2), 225-237.

Pas, E.T.; Waasdorp, T.E.; Bradshaw, C.P. (2015). Examining contextual influences on classroom-based implementation of positive behavior support strategies: Findings from a randomized controlled effectiveness trial. Prevention Science, 16(8), 10961106.

Pedroso, R.T.; Abreu, S.; Kinoshita, R.T. (2015). Aprendizagens da intersetorialidade entre saúde e educação na prevenção do uso de álcool e outras drogas. Revista Textura (Ulbra), 17(33).

Pereira, A.P.; Paes, Â.; Sanchez, Z.M. (2016). Factors associated with the implementation of programs for drug abuse prevention in schools. Revista Saude Publica, 50, 44.

Rajan, S.; Basch, C.E. (2012). Fidelity of After-School Program Implementation Targeting Adolescent Youth: Identifying Successful Curricular and Programmatic Characteristics. Journal of school health, 82(4), 159-165. 
Rice P; Ezzy D. (2000). Qualitative Research Methods: A Health Focus. South Melbourne, VIC: Oxford University Press.

Ringwalt, C.L.; Vincus, A.; Ennett, S.; Johnson, R.; Rohrbach, L.A. (2004). Reasons for teachers' adaptation of substance use prevention curricula in schools with non-white student populations. Prevention Science, 5(1), 61-67.

Sanchez, Z.M.; Sanudo, A.; Andreoni, S.; Schneider, D.; Pereira, A.P.; Faggiano, F. (2016). Efficacy evaluation of the school program Unplugged for drug use prevention among Brazilian adolescents. BMC Public Health, 16(1), 1206.

Sanchez, Z.M.; Santos, M.G.; Pereira, A.P.; Nappo, S.A.; Carlini, E.A.; Carlini, C.M.; Martins, S.S. (2013). Childhood alcohol use may predict adolescent binge drinking: a multivariate analysis among adolescents in Brazil. J Pediatr, 163(2), 363-368.

Saunders, R.P.; Evans, M.H.; Joshi, P. (2005). Developing a process-evaluation plan for assessing health promotion program implementation: a how-to guide. Health Promotion Practice, 6(2), 134-147.

Spoth, R.; Greenberg, M.; Turrisi, R. (2008). Preventive interventions addressing underage drinking: state of the evidence and steps toward public health impact. Pediatrics, 121(4), 311-336.

Sussman, S.; Stacy, A.W.; Johnson, C.A.; Pentz, M.A.; Robertson, E. (2004). A transdisciplinary focus on drug abuse prevention: an introduction. Subst Use Misuse, 39(10-12), 1441-1456.

Sy, A.; Glanz, K. (2008). Factors influencing teachers' implementation of an innovative tobacco prevention curriculum for multiethnic youth: Project SPLASH. The Journal of School Health, 78(5), 264273.

Thaker, S.; Steckler, A.; Sánchez, V.; Khatapoush, S.; Rose, J.; Hallfors, D.D. (2008). Program characteristics and organizational factors affecting the implementation of a school-based indicated prevention program. Health Education Research, 23(2), 238-248.

Vadrucci, S.; Vigna-Taglianti, F.D.; van der Kreeft, P.; Vassara, M.; Scatigna, M.; Faggiano, F.; Group, E.D.S. (2015). The theoretical model of the school-based prevention programme Unplugged. Global Health Promotion, 10.

Vigna-Taglianti, F.; Vadrucci, S.; Faggiano, F.; Burkhart, G.; Siliquini, R.; Galanti, M.R.; Group, E.D.S. (2009). Is universal prevention against youths' substance misuse really universal? Gender-specific effects in the EU-Dap school-based prevention trial. $J$ Epidemiol Community Health, 63(9), 722-728. 


\section{Sobre as autoras}

Pollyanna Fausta Pimentel de Medeiros (pollypimenta@yahoo.com)

Assistente social e mestre em Saúde Coletiva pela Universidade Federal de Pernambuco (UFPE). Doutora em Saúde Coletiva pelo Programa de Medicina Preventiva da Universidade Federal de São Paulo (UNIFESP). Membro do Grupo de Estudos em Álcool e outras Drogas (GEAD/ UFPE). Universidade Federal de São Paulo. São Paulo-SP. https://orcid.org/0000-0003-1679-5330 - Contribuição no artigo: concepção, análise, interpretação dos dados e redação final.

Ana Paula Dias Pereira (pauladias24@yahoo.com.br)

Doutoranda do programa de Saúde Coletiva - UNIFESP. Mestre em ciências pelo programa de Saúde Coletiva do Departamento de Medicina Preventiva da Universidade Federal de São Paulo - UNIFESP (2014). Possui graduação em Psicologia pela Universidade de Santo Amaro (2002) e Pós Graduação (Lato Sensu) em Psicologia Hospitalar pela Universidade de Santo Amaro (2004). Universidade Federal de São Paulo. São Paulo-SP. http://orcid.org/0000-0001-9944-4004 - Contribuição no artigo: análise dos dados e redação final.

Daniela Ribeiro Schneider (danischneiderpsi@gmail.com)

Graduada em Psicologia pela Universidade Federal de Santa Catarina (1987), Mestrado em Educação pela Universidade Federal de Santa Catarina (1993), Doutorado em Psicologia (Psicologia Clínica) pela Pontifícia Universidade Católica de São Paulo (2002) e Pós-Doutorado pela Universidade de Valencia - Espanha (2012). Atualmente é prof. associado III da Universidade Federal de Santa Catarina. Florianópolis- SC. http:/l orcid.org/0000-0002-2936-6503 - Contribuição no artigo: revisão crítica do manuscrito

Zila M. Sanchez (zila.sanchez@gmail.com)

É professora do Departamento de Medicina Preventiva, Disciplina de Epidemiologia, da Universidade Federal de São Paulo (UNIFESP). Possui graduação em Farmácia-Bioquímica pela USP, mestrado, doutorado e pós-doutorado em Ciências pela UNIFESP. Realizou doutoramentosanduíche na Universidade Autônoma de Barcelona, em Antropologia Social e Cultural e pós-doutorado no Departamento de Epidemiologia da Michigan State University, nos EUA, com cursos de epidemiologia (especialmente análises de dados epidemiológicos) realizados na Johns Hopkins Bloomberg School of Public Health e Columbia University. É professora-orientadora do programa de Pós-graduação em Saúde Coletiva da UNIFESP e pesquisadora do Centro Brasileiro de Informações Sobre Drogas Psicotrópicas (CEBRID/UNIFESP). Universidade Federal de São Paulo. São Paulo-SP. http://orcid.org/0000-0002-7427-7956 - Contribuição no artigo: concepção e desenho da pesquisa e revisão crítica do manuscrito. unrestricted use, distribution and reproduction in any medium, provided the original article is properly cited. 